\title{
Natural Convection of a 2D Boussinesq Fluid does NOT Maximise Entropy Production
}

\author{
Stuart Bartlett and Seth Bullock \\ Institute for Complex Systems Simulation \\ University of Southampton \\ Southampton, SO17 1BJ, UK \\ S.J.Bartlett@soton.ac.uk; stuart.bartlett@epfl.ch \\ (Dated: August 26, 2014)
}

\begin{abstract}
Rayleigh Bénard convection is a canonical example of spontaneous pattern formation in a nonequilibrium system. It has been the subject of considerable theoretical and experimental study, primarily for systems with constant (temperature or heat flux) boundary conditions. In this investigation, we have explored the behaviour of a convecting fluid system with negative feedback boundary conditions. At the upper and lower system boundaries, the inward heat flux is defined such that it is a decreasing function of the boundary temperature. Thus the system's heat transport is not constrained in the same manner that it is in the constant temperature or constant flux cases. It has been suggested that the entropy production rate (which has a characteristic peak at intermediate heat flux values) might apply as a selection rule for such a system. In this work, we demonstrate with Lattice Boltzmann simulations that entropy production maximisation does not dictate the steady-state of this system, despite its success in other, somewhat similar scenarios. Instead we will show that the same scaling law of dimensionless variables found for constant boundary conditions also applies to this system.
\end{abstract}

PACS numbers:

\section{INTRODUCTION}

Natural convection (NC) enjoys a prestigious position as an exemplar of pattern formation in a non-equilibrium system. It is remarkable that such a simple system as a differentially heated layer of fluid can exhibit such an array of coherent and beautiful fluid structures. The phenomenon of buoyancy-induced heat transfer has been the subject of intense experimental and theoretical study since the pioneering days of Lord Rayleigh and Henri Bénard, after which the most well-known type of NC is named. The bifurcation of a heated fluid from the diffusive state into a sustained pattern of convection rolls has long been recognised as a manifestation of the system's attempts to return itself to equilibrium. In fact, some of the most well known, early analytical work on the problem suggested that convecting fluids adjust their flows so as to maximise the rate of heat flux 1 13 .

One of the interesting characteristics of NC is that it has a highly non-linear force-flux response and an ongoing subject of research is to establish the nature of the scaling between the dimensionless forcing parameter, the Rayleigh number Ra and the ratio of total to diffusive heat flux, the Nusselt number $\mathrm{Nu}$ (see Eqs. 5 and 6 for definitions).

A range of analyses have suggested values for the scaling exponent of the $\mathrm{Ra}-\mathrm{Nu}$ relationship but recent high resolution numerical studies appear to be converging towards a consensus for certain values of the Prandtl number 4, 5. The majority of NC investigations applied fixed temperature (perfectly conducting) boundaries. This boundary condition (BC) has analytic and experimental utility (although real materials can of course never be perfectly conducting), and has long been used as a benchmark experimental setup. Researchers have also investigated other BCs such as constant boundary heat flux [5].

With fixed temperature boundaries, if all parameters are held constant, the system has a single macroscopic degree of freedom: the average heat flux. Since the boundary temperatures are fixed, higher heat fluxes correspond to higher rates of entropy production and vice versa.

With fixed flux boundaries, when all parameters are held constant, the average temperature difference across the system becomes the single free variable. Since entropy production is proportional to the difference of inverse temperatures, it increases monotonically with temperature difference (although not linearly).

As well as looking at state selection at constant parameter values, one can also adjust the properties of the fluid to assess changes in steady-state variables. It is well known that there is a monotonic increase in Nusselt number as the Rayleigh number is increased (as the convective resistance of the fluid is decreased). Decreasing the convective resistance can be achieved in several ways including increasing the system size, decreasing the viscosity, decreasing the thermal diffusivity or any combination of parameter changes which increases Ra.

For fixed temperature systems, if the thermal diffusivity is held constant, then any decrease in the convective resistance will produce an increase in the system's average heat flux (and an increase in entropy production).

With fixed heat flux, there is also a monotonic increase in Nusselt number as the convective resistance decreases. If the thermal diffusivity is held constant in this case, any decrease in convective resistance leads to a decrease 
in steady-state boundary temperature difference (and a decrease in entropy production).

So fixed BCs constrain the system to adjust either its heat flux or temperature differential. However there are alternative BCs, which prescribe neither the heat flux nor the temperature difference. Inspired by work on the theory of Maximum Entropy Production (MEP) [8, 9], we have simulated NC under such conditions, so-called negative feedback BCs [10].

Each boundary receives a constant inward heat flux but emits heat at a rate that is proportional to the instantaneous boundary temperature. There are thus several avenues through which the system can channel the thermal forcing that it receives. With a very small flux through the fluid, the system would experience a large net temperature difference. Conversely if the system was to transport a relatively large quantity of heat, the temperature difference would become small. In either case the balance of heat release between the two boundaries changes markedly as does the entropy produced as a result of the heat transfer.

A system with constant temperature BCs only produces more entropy as its heat flux increases. In contrast, a constant flux system shows a lower entropy production as its mean temperature difference falls. However with negative feedback BCs, rather than monotonic changes, there is a peak in entropy production at intermediate heat flux values. At the macroscopic level (and ignoring internal dynamics), such a system is essentially identical to the original box models used to approximate atmospheric heat transport when the theory of MEP was first proposed [11. Those original analyses have inspired a plethora of investigations into the relevance, applicability and theoretical underpinnings of MEP [8, 12,15]. Many researchers have observed what appears to be MEP in a variety of systems, however, a universally accepted derivation of MEP is still lacking. Hence it currently cannot be regarded as a unifying principle of non-equilibrium thermodynamics [16].

A convecting fluid system with negative feedback BCs represents an ideal test case for this theory, since it clearly has the ability to exhibit states of MEP. As well as a test of MEP, elucidating the temperature and flux response of a system with such flexible BCs is also highly desirable since one can then compare the scaling of the dimensionless groups $\mathrm{Ra}$ and $\mathrm{Nu}$, with those of fixed temperature and fixed flux systems. We have used a Lattice Boltzmann based algorithm to numerically resolve the hydrodynamic and thermodynamic characteristics of such a system for a range of fluid properties. In this paper we report the results of those simulations.

The paper is arranged as follows. In the following section we define our model system, paying close attention to the details of the BCs and steady-state transport properties. We will see that when the energy balance equations are de-dimensionalised, the system has only a single macroscopic degree of freedom. We then outline how it was simulated using the Lattice Boltzmann Method
(LBM). The next section summarises the results of our numerical investigations. They lead us to conclude that MEP does not represent a steady-state attractor for $\mathrm{NC}$ systems with negative feedback BCs. Finally, we discuss our findings with regard to the relevance and applicability of MEP for fluid heat transfer and suggest directions for future research.

\section{MODEL SYSTEM}

Before defining the system to be modelled, it is instructive to take a brief look at the equations of motion for an incompressible thermal fluid. It is assumed to be Boussinesq in character. This means that its physical properties remain constant and variations in density are assumed to have a negligible effect on the flow except with regard to the buoyancy-induced gravitational body force. Incompressibility leads to a simple form for the continuity equation,

$$
\nabla \cdot \mathbf{v}^{\prime}=0
$$

where $\mathbf{v}^{\prime}=u^{\prime} \hat{i}+w^{\prime} \hat{k}$ is the dimensional fluid velocity. Applying Newton's second law to a fluid parcel yields the momentum equation,

$$
\frac{\partial \mathbf{v}^{\prime}}{\partial t}+\mathbf{v}^{\prime} \cdot \hat{\nabla} \mathbf{v}^{\prime}+\frac{1}{\rho} \hat{\nabla} P^{\prime}=\nu \hat{\nabla}^{2} \mathbf{v}^{\prime}+B g_{0}\left(T^{\prime}-T_{0}^{\prime}\right) \hat{k}
$$

where $P^{\prime}$ is the pressure, $B$ is the coefficient of thermal expansion, $g_{0}$ is the strength of gravitational acceleration and $T^{\prime}$ is the temperature. Defining a set of characteristic scales allows us to de-dimensionalise this equation. We can choose a characteristic length $\delta$, time $\delta^{2} / \chi$ and temperature $\Delta T$ (temperature difference across the system). We thus find,

$$
\frac{\partial \mathbf{v}}{\partial t}+\mathbf{v} \cdot \nabla \mathbf{v}+\nabla P=\frac{\nu}{\chi} \nabla^{2} \mathbf{v}+\frac{\nu}{\chi} \frac{B g_{0} \Delta T \delta^{3}}{\nu \chi} T^{*} \hat{k}
$$

with dimensionless variables $\mathbf{v}, T^{*}$ and $P$. We see that the flow characteristics are defined by two dimensionless groups,

$$
\operatorname{Pr}=\frac{\nu}{\chi}
$$

and

$$
R a=\frac{B g_{0} \Delta T \delta^{3}}{\nu \chi}
$$

The Prandtl number is simply the ratio of momentum to thermal diffusivity and the Rayleigh number defines the dimensionless driving force (in this work any reference to convective resistance simply means the inverse of Rayleigh number). Any two flows with equal Ra and Pr, will be fluid dynamically equivalent.

Note that for a system with fixed temperature BCs, Ra is fixed by the BCs. For a fixed flux system, Ra becomes 
a variable which depends on the steady-state transport properties. Similarly, negative feedback BCs do not fix $\Delta T$ and thus $\mathrm{Ra}$ is a variable rather than a parameter for such systems.

Finally, let us define the ratio of total to diffusive heat flux, the Nusselt number:

$$
N u=\frac{J_{a b}}{\chi \Delta T / \delta}
$$

where $J_{a b}$ is the heat flux. The free variable for fixed temperature systems is the heat flux $J_{a b}$. For fixed flux systems $J_{a b}$ is defined by the BCs and thus $\Delta T$ becomes the free variable. For negative feedback BCs neither $J_{a b}$ nor $\Delta T$ are fixed. However, we will see below that these systems also have only a single macroscopic degree of freedom.

Let us now define all the details of the negative feedback system. We assume we are dealing with a twodimensional fluid system enclosed between two solid boundaries. In the horizontal direction the system's boundaries are periodic and the aspect ratio is 2 . The upper and lower boundaries receive inward heat fluxes $J_{i n, b}$ and $J_{i n, a}$ respectively, and are also able to radiate heat away. The outward fluxes have the form $J_{\text {out }, i}=\beta T_{i}$ where $i \in\{a, b\}$ and $\beta$ is a parameter. Fig. 1 shows a schematic of the system. Heat flows through it by a com-

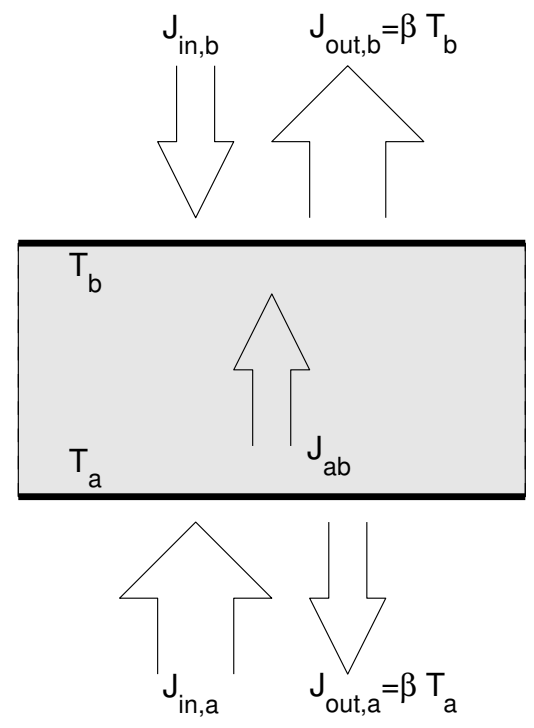

FIG. 1: Model system diagram showing the various heat fluxes, which comprise the boundary energy balances. The solid walls enforce the no-slip velocity condition.

bination of diffusion and convection. As mentioned previously, simple box models with similar BCs have been used to approximate atmospheric heat transport [13, 17] and have been explored analytically [see, e.g., 8]. If we ignore the internal details of the system and regard it as a black box that has reached a state of dynamic equilibrium, we can write the energy balance at the two boundaries,

$$
\begin{aligned}
& J_{i n, a}-J_{a b}-\beta T_{a}=0 \\
& J_{i n, b}+J_{a b}-\beta T_{b}=0 .
\end{aligned}
$$

Adding these two equations yields

$$
J_{i n, a}+J_{i n, b}-\beta\left(T_{a}+T_{b}\right)=0
$$

If we write the two temperatures in the form $T_{a}=T_{0}+$ $\Delta T / 2, T_{b}=T_{0}-\Delta T / 2$, we can use Eq. 9 to find an expression for the system's average temperature:

$$
T_{0}=\frac{J_{i n, a}+J_{i n, b}}{2 \beta} .
$$

To find an expression in terms of $\Delta T$ we can subtract Eq. 8 from Eq. 7

$$
J_{i n, a}-J_{i n, b}-2 J_{a b}=\beta \Delta T .
$$

We see here that if the temperature difference is known, then the heat flux is determined since the other terms are boundary parameters. So in fact this system, like those with fixed $\mathrm{BCs}$, has only one macroscopic degree of freedom.

At this point it is useful to de-dimensionalise Equation 11 to uncover the dependance of the system's steadystate upon the various parameters. Let us define the dimensionless flux parameter $\bar{\beta}=\frac{\delta}{\chi} \beta$ and flux difference parameter, $R_{J}=\frac{B g_{0} \delta^{4}}{\nu \chi^{2}}\left[J_{i n, a}-J_{i n, b}\right]$. If we now re-arrange Equation 11 and perform several stages of algebraic manipulation, we find

$$
J_{a b}=\frac{1}{2} \frac{\chi \Delta T}{\delta}\left(\frac{R_{J}}{R a}-\bar{\beta}\right)
$$

and thus,

$$
N u=\frac{1}{2}\left(\frac{R_{J}}{R a}-\bar{\beta}\right) .
$$

We see that when the fluid and boundary parameters are fixed, the Nusselt number depends only upon the steadystate temperature difference (the single degree of freedom of the system). Note also that both fluid and boundary parameters influence the Nusselt number.

Let us now turn to the entropy production rate $\sigma$. Noting the dimensions of $\beta$, the dimensionless entropy production can be defined as $\bar{\sigma}=\sigma / \beta$. We must also define a dimensionless mean temperature, $\overline{T_{0}}=\frac{B g_{0} T_{0} \delta^{3}}{\nu \chi}$. Once more some algebraic manipulation yields an expression for the dimensionless entropy production,

$$
\begin{array}{r}
\bar{\sigma}=\frac{J_{a b}}{\beta}\left(\frac{1}{T_{b}}-\frac{1}{T_{a}}\right) \\
=\frac{1}{2 \beta}\left(\frac{J_{i n, a}-J_{i n, b}}{\Delta T}-\beta\right) \frac{4 \Delta T^{2}}{4 T_{0}^{2}-\Delta T^{2}} \\
=\frac{1}{2 \bar{\beta}}\left(\frac{R_{J}}{R a}-\bar{\beta}\right) \frac{4 R a^{2}}{4 \bar{T}_{0}^{2}-R a^{2}}
\end{array}
$$


We see again that when reduced to a dimensionless form, the steady-state of the system is a function of a single variable (the temperature difference $\Delta T$, within Ra). Furthermore, the dimensionless entropy production depends only upon boundary parameters and $\Delta T$, as shown in Equation 15.

In this investigation we used a single set of boundary parameters, $\left(J_{i n, a}=0.1, J_{i n, b}=0.01, \beta=0.1\right)$ and only varied the physical properties of the fluid. The state selection MEP principle would suggest that for a given set of boundary and fluid parameters, the steady-state of the system should adjust to one in which $\bar{\sigma}$ is maximised with respect to $\Delta T$. In Figure 2, the boundary temperatures and dimensionless entropy production are plotted as a function of the normalised heat flux. Temperature

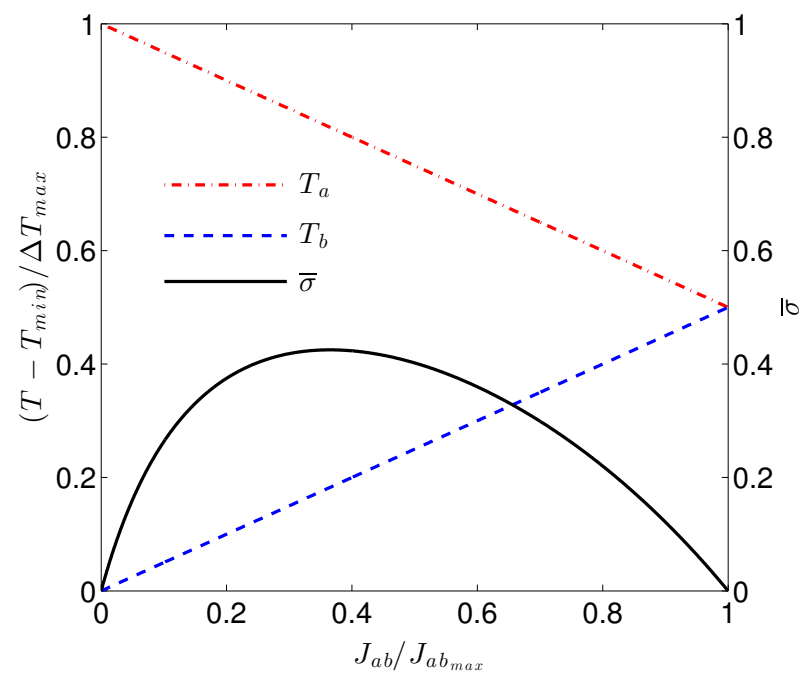

FIG. 2: (Color online) Macroscopic, steady-state properties of the model system. Boundary temperatures $T_{a}$ (red dot-dash line) and $T_{b}$ (blue dashed line), and dimensionless entropy production (solid black line) are plotted as a function of the normalised heat flux.

values are normalised using the maximum temperature difference $\Delta T_{\max }=\left(J_{i n, a}-J_{i n, b}\right) / \beta$ (this occurs when the heat flux $\left.J_{a b}=0\right)$. Heat flux values are normalised by the relevant maximum: $J_{a b_{\max }}=\left(J_{i n, a}-J_{i n, b}\right) / 2$ (this occurs when the temperature difference $\Delta T=0)$.

Figure 2 shows that $\bar{\sigma}$ has a single peak at intermediate values of heat flux. This contrasts with the case of fixed $\mathrm{BCs}$, in which changes in the single free variable cause only monotonic increases or decreases in entropy production. Thus a negative feedback system permits an ideal test of the state selection MEP principle for $\mathrm{NC}$ heat transfer.

Note however that $\bar{\sigma}$ is not a function of any fluid parameters (see Equation 15). Thus for MEP to be a steady-state attractor, different fluids would all have to adhere to the same value of $J_{a b}$ in steady-state.

As mentioned in the introduction, in recent years there has been a great deal of discussion concerning MEP since it has shown considerable utility for predicting the steady-state behaviour of certain non-equilibrium systems including crystal growth [18, 19, electrical current flow 20, ecosystems 21] [also see articles within 22 and plant functional optimisation 23. Inspired by these successes, it was hoped that MEP may in fact be a unifying principle of non-equilibrium thermodynamics. Its application to models of heat and momentum transport through simple flow systems have shown reasonable quantitative agreement with experimental results.

In particular, the work of Ozawa et al. 17] predicted a scaling law of $N u \sim R a^{1 / 3}$. However the dependance of $\mathrm{Nu}$ on $\mathrm{Ra}$ for $\mathrm{NC}$ systems is still not fully understood. It is known to vary with $\operatorname{Pr}$ and a more recent numerical study found that $N u \sim R a^{0.285}$ for turbulent convection with $\operatorname{Pr}=1$ [5]. Clearly the MEP prediction does not tell the entire story. However it has enjoyed success in a related area: the analysis of atmospheric heat transport [11 13. Ozawa et al. [13] found that certain transport properties of the atmospheric circulation of several planetary bodies including Earth can be reliably predicted using a simple application of the principle of MEP. Kleidon [8] also suggested that in a simple convective fluid system, the characteristic peak seen in Fig. 2 will define the steady-state heat flux of the system.

This was part of the motivation for this investigation, as a test of MEP for a simple non-equilibrium system. As noted above, the entropy production peak occurs without any incorporation of the particular physical properties of the fluid. It comes purely from an analysis of the steady-state energy balance. Surely a more viscous fluid would adopt a different steady-state heat flux than one that was less viscous, for example. In Sec. IV] we show exactly how our system responds in this respect. First we briefly describe the numerical implementation of the above model. Those not interested in the details of the simulation technique can skip straight to Sec. IV.

\section{IMPLEMENTATION WITH THE LATTICE BOLTZMANN METHOD}

The numerical model used in this investigation is the two-dimensional D2Q9 (2 dimensions and 9 discrete particle velocities) Thermal Lattice Boltzmann model (TLBM) of Peng et al. 24, which is a simplified version of the model of He et al. 25]. This is essentially a kineticbased mesoscopic technique which numerically solves a discretised form of the Boltzmann equation, making use of the BGK collision operator [26] to simplify the collision term. The method stands in stark contrast to traditional computational fluid dynamics (CFD) techniques, which typically begin with continuum equations of motion for fluid variables (the Navier-Stokes equations) and seek solutions using numerical techniques.

The LBM, however, begins from a kinetic description of the fluid, and the fluid equations of motion actually emerge at macroscopic length scales from the microscopic 
dynamics using a suitable Chapman-Enskog expansion [see 27, for a review]. While most LBMs are isothermal, the version we have made use of simulates the internal energy field of the fluid as an additional set of distribution functions on the same discrete lattice as the fluid mass. Heat is thus modelled as a passive scalar being advected by the fluid and not interacting with it except in the form of a buoyancy-driven body force caused by temperature-induced density variations in the presence of a gravitational field. Note that viscous dissipation is not included in this version and the fluid is assumed to be Boussinesq in character.

This form of TLBM has been repeatedly shown to be capable of reproducing experimental results for $\mathrm{NC}$ of a single-phase fluid. For details of those results and a complete description of the model, please see Peng et al. 24, He et al. 25, Dixit and Babu 28].

Here we describe the unique BCs employed for this study. For the velocity distribution functions, we used the halfway wall bounce-back method. We found that using more complicated BCs made no significant difference to the results. For the internal energy distributions there are two alternatives for applying the relevant boundary heat fluxes at each integration step. The first method, which we will refer to as the vari method involves a simple point-by-point application of the BC. For the boundary grid nodes, after the bounce-back stage of the algorithm and before the collision step, the three inwardpointing internal energy distribution functions underwent the following forcing step:

$$
g_{j, k}^{\prime}=g_{j, k}+\frac{6 \omega_{j}}{W}\left(J_{i n_{i}}-\beta T_{i, k}\right)
$$

where $j$ indexes the distribution function (for a lower boundary, $j \in\{2,5,6\}$ and for an upper boundary, $j \in$ $\{4,7,8\}), i \in\{a, b\}$ denotes the upper or lower boundary, $k$ denotes the grid node $\left(T_{i, k}\right.$ is the temperature of node $k$ on the boundary $i$ calculated after streaming and bounceback), $\omega_{j}$ is the distribution function weight and $W$ is the number of grid nodes in the horizontal direction. Note that $1 /\left(\omega_{4}+\omega_{7}+\omega_{8}\right)=1 /\left(\omega_{2}+\omega_{5}+\omega_{6}\right)=6$. With this form of $\mathrm{BC}$ the temperature profiles on the boundaries are not horizontally uniform.

The second BC, which we denote the uni method, assumes that the boundaries of the system have perfect thermal conductivity in the horizontal direction such that they always exhibit a horizontally uniform temperature profile. The first stage is to calculate the mean boundary temperature $\overline{T_{i}}$ after streaming and bounce-back. The total heat flux into the boundary can then be calculated:

$$
J_{\text {tot }_{i}}=J_{i n_{i}}-\beta \overline{T_{i}}
$$

We will require the expression for the total internal energy at grid node $k$,

$$
\rho_{k} \epsilon_{k}=\sum_{j=0}^{8} g_{j, k}
$$

where $\rho_{k}$ and $\epsilon_{k}$ are the mass and internal energy densities of grid node $k$. Summing this over the entire length of the boundary gives an expression for the total boundary internal energy:

$$
E=\sum_{k=0}^{W-1} \rho_{k} \epsilon_{k}
$$

We can now calculate the new boundary internal energy after the heat flux step:

$$
\begin{array}{r}
E^{\prime}=E+J_{\text {tot }_{i}} \\
=J_{i n_{i}}-\beta \overline{T_{i}}+\sum_{k=0}^{W-1} \rho_{k} \epsilon_{k}
\end{array}
$$

It only remains to calculate the new boundary internal energy density $\epsilon^{\prime}$ resulting from this inward flux of heat. Remember that we wish to distribute the incoming energy such that every boundary grid node will have this new temperature $\left(\epsilon^{\prime}\right.$ does not depend on the node index $k)$. We make use of Eq. 20 .

$$
\begin{array}{r}
\sum_{k=0}^{W-1} \rho_{k} \epsilon^{\prime}=E^{\prime}=J_{i n_{i}}-\beta \overline{T_{i}}+\sum_{k=0}^{W-1} \rho_{k} \epsilon_{k} \\
\epsilon^{\prime} \sum_{k=0}^{W-1} \rho_{k}=J_{i n_{i}}-\beta \overline{T_{i}}+\sum_{k=0}^{W-1} \rho_{k} \epsilon_{k} \\
\epsilon^{\prime}=\frac{J_{i n_{i}}-\beta \overline{T_{i}}+\sum_{k=0}^{W-1} \rho_{k} \epsilon_{k}}{\sum_{k=0}^{W-1} \rho_{k}}
\end{array}
$$

The inward-pointing internal energy distributions can then be modified before the collision step according to:

$$
g_{j, k}^{\prime}=g_{j, k}+6 \omega_{j} \rho_{k}\left(\epsilon^{\prime}-\epsilon_{k}\right)
$$

Other than the above BC modifications, our model was identical to that described in Peng et al. 24. All simulations were initialised in a state of zero fluid velocity at the mean temperature (Eq. 10), with a uniform internal energy distribution and a small degree of random noise. Runs were concluded when the system's key variables (boundary temperatures, Rayleigh and Nusselt numbers) showed no further variation with time. In the more turbulent cases $\left(R a>10^{6}\right)$, in which the variables showed no sign of becoming stationary, but instead showed oscillatory behaviour, a long time average was taken.

Table I shows the various grid sizes and relaxation parameters used in this investigation. Note that the internal energy relaxation parameter can be calculated from the fluid relaxation parameter due to the $\operatorname{Pr}=1$ condition.

\section{RESULTS}

We now summarise the findings of our numerical experiments. For the sake of comparison we also simulated 
TABLE I: Lattice Boltzmann Model parameters

\begin{tabular}{lcc}
\hline \hline $\mathrm{Ra}$ & $\tau_{\nu}$ & $\mathrm{W}$ \\
\hline $5.4 \times 10^{2}$ & 0.76 & 128 \\
$1.1 \times 10^{3}$ & 0.70 & 128 \\
$2.1 \times 10^{3}$ & 0.81 & 256 \\
$4.0 \times 10^{3}$ & 0.72 & 256 \\
$1.1 \times 10^{4}$ & 0.72 & 400 \\
$2.0 \times 10^{4}$ & 0.67 & 400 \\
$4.6 \times 10^{4}$ & 0.62 & 400 \\
$1.3 \times 10^{5}$ & 0.60 & 512 \\
$4.3 \times 10^{5}$ & 0.56 & 512 \\
$2.0 \times 10^{6}$ & 0.92 & 6144 \\
$9.0 \times 10^{6}$ & 0.72 & 6144 \\
$3.8 \times 10^{7}$ & 0.62 & 6144 \\
$1.0 \times 10^{8}$ & 0.58 & 6144 \\
\hline \hline
\end{tabular}

the same system with fixed temperature and fixed flux BCs. The scaling of the two key dimensionless groups, $\mathrm{Ra}$ and $\mathrm{Nu}$ for the fixed $\mathrm{BC}$ systems is shown in Fig. $3(\mathrm{a})$

This figure shows essentially identical behaviour to the analogous graph in the work of Johnston and Doering [5], implying that our TLBM can simulate NC systems up to $R a=10^{8}$ with reasonable accuracy. To simulate even more turbulent systems would have required considerable computing power and time or the use of non-uniform grid meshes. In this work however, we restricted ourselves to $R a \leq 10^{8}$.

We now move on to simulations with negative feedback BCs, illustrated in Fig. 3(b). The scaling of $\mathrm{Ra}$ and $\mathrm{Nu}$ appears to be identical to the fixed temperature and fixed flux data, and adheres to the same scaling law proposed by Johnston and Doering [5] when $R a \geq 10^{7}$. Notice also that at lower Ra the simulations which permitted a nonuniform boundary temperature profile (vari) were able to achieve an augmented Nusselt number compared to those with uniform boundary temperatures (uni). This is similar to the slight differences between fixed flux and fixed temperature systems over the same range of Ra values. The fixed flux BCs also allowed a slightly higher $\mathrm{Nu}$ since the convection rolls penetrated into the region which would normally be occupied by boundary layers in the fixed temperature systems.

Having seen that in terms of conventional dimensionless measures, negative feedback systems behave identically to those with fixed BCs, we can now consider entropy production. In Fig. 4 the steady-state boundary temperature differences $\Delta T$ are plotted as a function of the heat flux $J_{a b}$, normalised by their relevant maxima.

Two conclusions can be drawn immediately: 1) The heat flux values are all significantly larger than the flux value corresponding to MEP and 2) There is considerable spread in the heat flux values, caused by the variation in fluid properties between the different simulations. So for an individual system with fixed parameters, the MEP state does not appear to be any kind of attractor. Fur-

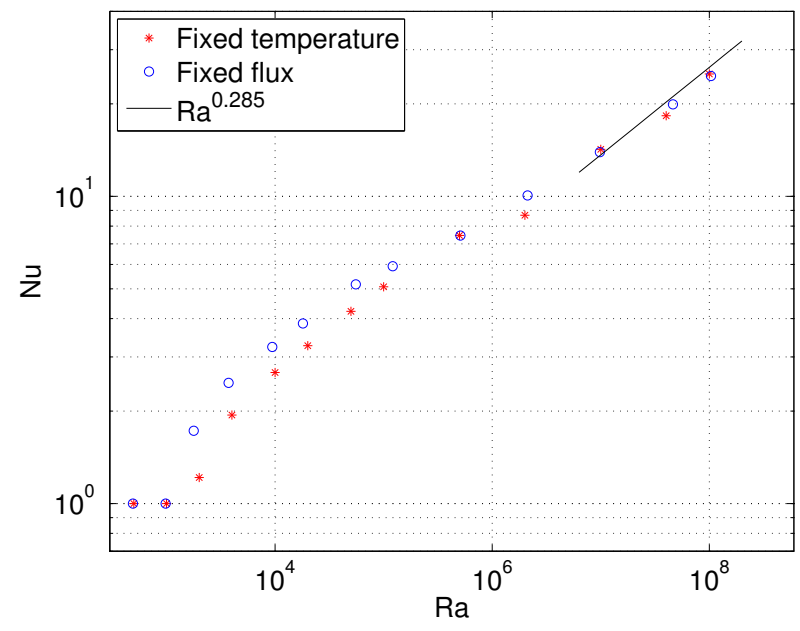

(a)

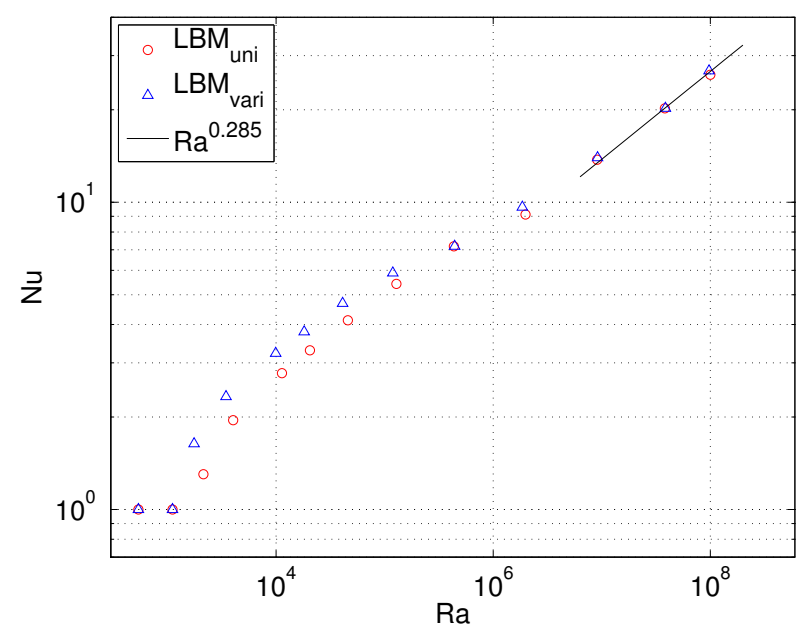

(b)

FIG. 3: (Color online) Ratio of total to diffusive heat flux $\mathrm{Nu}$ as a function of dimensionless thermal driving force Ra for $\mathrm{NC}$ systems with a) fixed temperature and fixed heat flux BCs and b) negative feedback BCs. Red asterisks correspond to fixed temperature BCs, blue circles to fixed flux BCs, red circles to negative feedback systems with uniform boundary temperature profiles and blue triangles to negative feedback systems with a variable boundary temperature profile. The solid black line shows the empirical scaling law $N u \approx 0.138 R a^{0.285}$ due to Johnston and Doering [5].

thermore when the fluid parameters are varied, there is a concomitant variation in the steady-state properties.

Note that the points also include simulations below the critical Rayleigh number, in which the diffusive state is the most stable and there is no convective heat transport. Even such low heat fluxes as these are still much greater than the MEP value.

Finally, Fig. 5 displays the temperature field of a negative feedback simulation. Streamlines are plotted to illustrate the structure of the thermally-induced flow. 


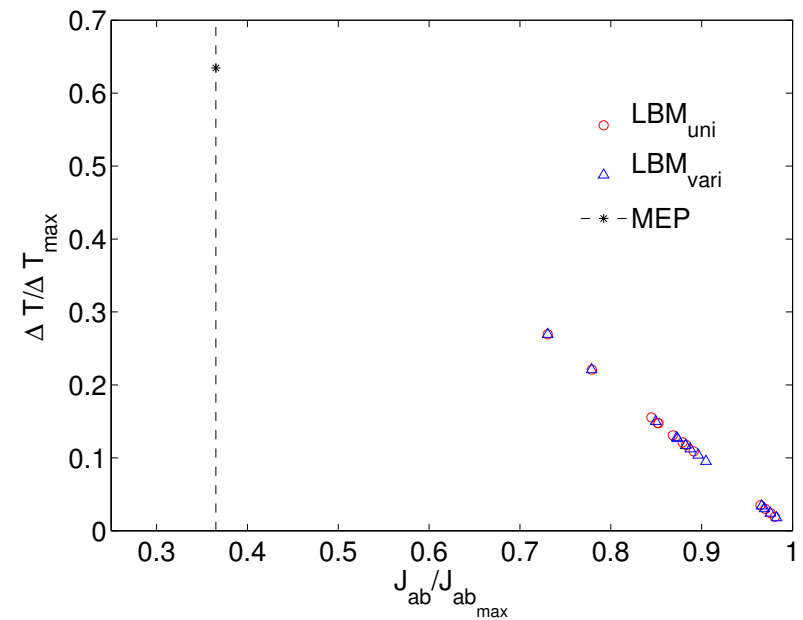

FIG. 4: (Color online) steady-state temperature difference as a function of total heat flux for TLBM simulations with negative feedback BCs. Red circles and blue triangles show results from the uni and vari simulations respectively. The black dotted line shows the heat flux value corresponding to a state of maximum entropy production and the black asterisk shows the corresponding value of the temperature difference.

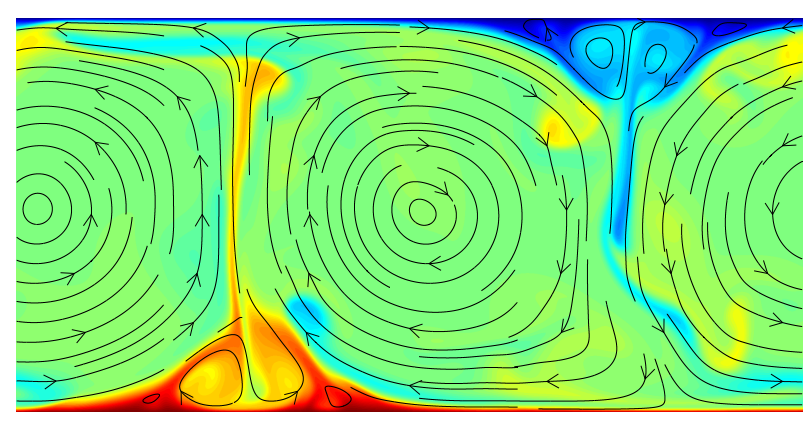

FIG. 5: (Color online) Temperature field of a natural convection fluid system with negative feedback BCs. This example is a uni system with $R a \approx 10^{8}$. Streamlines show the two principal convection rolls.

\section{CONCLUSIONS}

The numerical results of this work compel us to think critically about the basis and applicability of the principle of MEP, specifically its use for predicting the transport properties of non-equilibrium fluid systems. First we should consider the fact that in terms of dimensionless variables, $\mathrm{NC}$ flows behave identically no matter whether the BCs are fixed or allow some degree of feedback. The results re-enforce the natural intuition that in steadystate, a fluid system should be indifferent to the manner in which the BCs are prescribed.

There doesn't seem to be any reason why the system should be sensitive to entropy production, or why the MEP state should be attractive. The steady-state condition implies constant boundary temperatures and heat flux, and whether that heat flux is provided as a constraint or whether it emerges in response to a fixed temperature difference or a more complicated set of conditions, should have little impact on the internal flow configuration.

As we have seen there are some subtle differences at low Ra between fixed flux and fixed temperature systems, and between negative feedback systems with uniform and variable boundary temperature profiles. However such differences disappear when the boundaries are allowed to be perfectly conducting in the horizontal direction.

The principle of MEP has enjoyed considerable success when applied to simple models of atmospheric circulation as well as other non-equilibrium systems. However, there has also been criticism of the principle since it has no rigorous theoretical basis. In fact some early proponents of MEP have since changed position on the philosophical role of the principle (e.g. compare the work of 29 to 30 ). Furthermore, when it comes to fluid dynamical systems, entropy minimisation, as well as maximisation, has been observed [31, 32. Which extremum emerges seems to depend in a non-trivial way upon the BCs and particular flow being studied.

Note that there are several different forms that entropy maximisation can take. In the case of negative feedback $\mathrm{BCs}$, it refers to the selection of a state corresponding to MEP (the peak in Figure 2), under conditions of fixed fluid and boundary parameters. There is nothing to stop a given system settling into a state of MEP, and yet in our numerical investigation, such behaviour was never observed.

For fixed BCs, MEP takes on a slightly different form. Since the boundary temperature difference or heat flux is fixed, the range of possible steady-states does not exhibit an entropy production peak such as that of negative feedback BCs. Instead the entropy production either increases or decreases monotonically as a function of the single free variable (either heat flux or temperature difference). Since there is no clear MEP state, in this context, MEP is often framed as a selection mechanism between the two modes of heat transfer: pure diffusion and diffusion plus convection 33.

When the Rayleigh number is greater than that of the bifurcation point $R a>R a_{c} \approx 1706$, the system adopts a convective state of fluid motion instead of the static, purely diffusive state. It is frequently assumed that there is some connection to the fact that the convective state has the higher entropy production 33. However, in a system with fixed flux $\mathrm{BCs}$, at $R a>R a_{c}$, the convective state actually has a lower entropy production. This results from the lower temperature differences exhibited at higher $\mathrm{Ra}$ (a fluid with lower convective resistance requires a smaller driving gradient to achieve the same heat flux). So entropy production also has no bearing on systems with fixed BCs.

The final point to discuss is the fact that all of our data points exhibited heat flux values far greater than that of 
MEP. It should be possible to observe the entire range of $J_{a b}$ and $\Delta T$ values simply making the relevant parameter adjustments. This could have been accomplished if we had relaxed our assumption of constant Prandtl number. We could have altered our fluid such that its viscosity was very high, and its thermal diffusivity was very low. This would have begun to effectively block off the heat transport channel of the fluid. At both boundaries, the majority of the incoming heat would have immediately been re-emitted.

An alternative way for exploring the entire range of normalised heat flux values would be to adjust the boundary parameter $\beta$. Increasing $\beta$ causes a proportionate decrease in steady-state $\Delta T$ values but does not affect $J_{a b_{\max }}$. Therefore the entropy production peak occurs at the same value of $J_{a b}$ for all values of $\beta$. Increasing this parameter is likely to have the effect of funnelling heat flux away from the fluid system (reducing $J_{a b}$ ). Instead much of the energy entering the system will be immediately re-emitted at the boundaries, having never entered the fluid region. We are currently performing these simulations with different $\beta$ values and the results of that work will be published in the near future.

In conclusion, we do not believe that MEP is a univer- sal law of nature. Its successes and relationship to other entropy extremum principles are yet to be fully elucidated. A fruitful and very useful further study would be to make a comprehensive assessment of all the applications of MEP and try to uncover underlying universalities and connections between large systems and small systems, physical systems and chemical or biological systems.

As well as an assessment of the principle of MEP, we have also built upon the work of Johnston and Doering [5], who established that at high Rayleigh numbers, NC flows show the same dimensionless behaviour whether fixed temperature or fixed flux BCs are applied. We have shown that even when neither the boundary fluxes nor temperatures are fixed, the same Ra-Nu scaling emerges. More complicated BCs are unlikely to yield any deviations from this general behaviour.

\section{ACKNOWLEDGEMENTS}

This work was supported by an EPSRC Doctoral Training Centre grant (EP/G03690X/1).
[1] Malkus, W. V. R. (1954a). Discrete transitions in turbulent convection. Proceedings of the Royal Society of London, Series A, 225:185-195.

[2] Malkus, W. V. R. (1954b). The heat transport and spectrum of thermal turbulence. Proceedings of the Royal Society of London, Series A, 225(1161):196-212.

[3] Malkus, W. V. R. and Veronis, G. (1958). Finite amplitude cellular convection. Journal of Fluid Mechanics, $4(03): 225-260$.

[4] Ahlers, G., Grossmann, S., and Lohse, D. (2009). Heat transfer and large scale dynamics in turbulent RayleighBénard convection. Rev. Mod. Phys., 81:503-537.

[5] Johnston, H. and Doering, C. R. (2009). Comparison of turbulent thermal convection between conditions of constant temperature and constant flux. Phys. Rev. Lett., 102:064501.

[6] Otero, J., Wittenberg, R. W., Worthin, R. A., and Doering, C. R. (2002). Bounds on Rayleigh Bénard convection with an imposed heat flux. Journal of Fluid Mechanics, 473:191-199.

[7] Verzicco, R. and Sreenivasan, K. R. (2008). A comparison of turbulent thermal convection between conditions of constant temperature and constant heat flux. Journal of Fluid Mechanics, 595:203-219.

[8] Kleidon, A. (2009). Nonequilibrium thermodynamics and maximum entropy production in the earth system. Naturwissenschaften, 96:653-677.

[9] Martyushev, L. and Seleznev, V. (2006). Maximum entropy production principle in physics, chemistry and biology. Physics Reports, 426(1):1 - 45 .

[10] Virgo, N. D. (2011). Thermodynamics and the structure of living systems. PhD thesis, University of Sussex.

[11] Paltridge, G. W. (1978). The steady-state format of global climate. Quarterly Journal of the Royal Meteorological Society, 104(442):927-945.

[12] O'Brien, D. M. and Stephens, G. L. (1995). Entropy and climate. ii: Simple models. Quarterly Journal of the Royal Meteorological Society, 121(527):1773-1796.

[13] Ozawa, H., Ohmura, A., Lorenz, R. D., and Pujol, T. (2003). The second law of thermodynamics and the global climate system: A review of the maximum entropy production principle. Reviews of Geophysics, 41(4).

[14] Kleidon, A. (2010). A basic introduction to the thermodynamics of the earth system far from equilibrium and maximum entropy production. Philosophical Transactions of the Royal Society of London, Series B, 365(1545):1303-1315.

[15] Dyke, J. and Kleidon, A. (2010). The maximum entropy production principle: Its theoretical foundations and applications to the earth system. Entropy, 12(3):613-630.

[16] Bruers, S. (2007). A discussion on maximum entropy production and information theory. Journal of Physics A: Mathematical and Theoretical, 40(27):7441.

[17] Ozawa, H., Shimokawa, S., and Sakuma, H. (2001). Thermodynamics of fluid turbulence: A unified approach to the maximum transport properties. Phys. Rev. E, 64:026303.

[18] Hill, A. (1990). Entropy production as the selection rule between different growth morphologies. Nature, 348:426428.

[19] Martyushev, L. and Axelrod, E. (2003). From dendrites and s-shaped growth curves to the maximum entropy production principle. Journal of Experimental and Theoretical Physics Letters, 78(8):476-479.

[20] Zupanović, P. and Juretić, D. (2004). Kirchhoffs loop law and the maximum entropy production principle. Phys. 
Rev. E, 70:056108.

[21] Vallino, J. J. (2010). Ecosystem biogeochemistry considered as a distributed metabolic network ordered by maximum entropy production. Philosophical Transactions of the Royal Society of London, Series B, 365(1545):14171427.

[22] Kleidon, A., Malhi, Y., and Cox, P. M. (2010). Maximum entropy production in environmental and ecological systems. Philosophical Transactions of the Royal Society of London, Series B, 365(1545):1297-1302.

[23] Dewar, R. C. (2010). Maximum entropy production and plant optimization theories. Philosophical Transactions of the Royal Society of London, Series B, 365(1545):1429-1435.

[24] Peng, Y., Shu, C., and Chew, Y. T. (2003). Simplified thermal Lattice Boltzmann model for incompressible thermal flows. Phys. Rev. E, 68:026701.

[25] He, X., Chen, S., and Doolen, G. D. (1998). A novel thermal model for the Lattice Boltzmann Method in incompressible limit. Journal of Computational Physics, 146(1):282 - 300 .

[26] Bhatnagar, P. L., Gross, E. P., and Krook, M. (1954). A model for collision processes in gases. i. small amplitude processes in charged and neutral one-component systems. Phys. Rev., 94:511-525.

[27] Chen, S. and Doolen, G. D. (1998). Lattice Boltzmann method for fluid flows. Annual Review of Fluid Mechan- ics, 30(1):329-364.

[28] Dixit, H. and Babu, V. (2006). Simulation of high rayleigh number natural convection in a square cavity using the lattice boltzmann method. International Journal of Heat and Mass Transfer, 49(3-4):727-739.

[29] Dewar, R. (2003). Information theory explanation of the fluctuation theorem, maximum entropy production and self-organized criticality in non-equilibrium stationary states. Journal of Physics A: Mathematical and General, 36(3):631.

[30] Dewar, R. C. (2009). Maximum entropy production as an inference algorithm that translates physical assumptions into macroscopic predictions: Don't shoot the messenger. Entropy, 11(4):931-944.

[31] Niven, R. (2010). Simultaneous extrema in the entropy production for steady-state fluid flow in parallel pipes. Journal of Non-Equilibrium Thermodynamics, 35(3):113.

[32] Paulus Jr., D. M. and Gaggioli, R. A. (2004). Some observations of entropy extrema in fluid flow. Energy, 29(1215):2487 - 2500 .

[33] Meysman, F. J. R. and Bruers, S. (2010) Ecosystem functioning and maximum entropy production: a quantitative test of hypotheses. Philosophical Transactions of the Royal Society of London, Series B, 365(1545):14051416. 